\title{
Thermal stability of magnetic states in submicron magnetic islands
}

\author{
S. Y. Liashko ${ }^{1,2}$, I. S. Lobanov ${ }^{1}$, V. M. Uzdin ${ }^{1,3}$, H. Jónsson ${ }^{2,4}$ \\ ${ }^{1}$ ITMO University, Kronverkskiy, 49, St. Petersburg, 197101, Russia \\ Kronverkskiy, 49, St. Petersburg, 197101, Russia \\ ${ }^{2}$ Science Institute and Faculty of Physical Sciences, University of Iceland \\ 107 Reykjavík, Iceland \\ ${ }^{3}$ St. Petersburg State University, St. Petersburg, 198504, Russia \\ ${ }^{4}$ Center for Nonlinear Studies, Los Alamos, NM 87545, USA \\ sergei.liashko@gmail.com, lobanov.igor@gmail.com, v_uzdin@mail.ru,hj@hi.is
}

PACS 75.75.-c, 75.75.Jn, 75.60.Jk

DOI 10.17586/2220-8054-2017-8-5-572-578

The lifetime of magnetic states in single domain micromagnetic islands is calculated within the harmonic approximation to transition state theory. Stable magnetic states, minimum energy paths between them and first order saddle points determining the activation energy are analyzed and visualized on two-dimensional energy surfaces. An analytical expression is derived for the pre-exponential factor in the Arrhenius rate expression for the reversal of the magnetic moment when the external field is directed either along the anisotropy axis or perpendicular to it.

Keywords: pre-exponential factor, magnetic islands, activation energy, rate theory, spin ice.

Received: 19 September 2017

Revised: 14 October 2017

\section{Introduction}

The stability of magnetic states with respect to thermal fluctuations and external perturbations is an important topic in fundamental science as well as for technological applications [1,2]. Thermal stability is a particularly important issue in the context of nanoscale information storage devices. The thermal stability of the magnetic states decreases as the size of such devices is reduced. Estimates of the rate of magnetic transitions are, therefore, important when designing such systems.

In this context, thermally-activated magnetic transitions are rare events on the time scale of oscillations of the magnetic moments, making direct simulations of spin dynamics an impractical way to estimate the lifetime. This separation of time scales, however, makes it possible to apply statistical approaches such as transition state theory (TST) [3] or Kramers theory [4]. The transitions are slow enough that a Boltzmann distribution is established and maintained in the initial state of the system. Within the harmonic approximation to TST (HTST) [5] and within Kramers theory, the activation energy of a transition is given by the energy difference between the local minimum on the energy surface corresponding to the initial state and the highest energy on the minimum energy path (MEP) connecting the initial and final state minima. The MEPs between minima are the transition paths of largest statistical weight and characterize the mechanism of the corresponding transitions. A maximum along an MEP corresponds to a first order saddle point on the energy surface and gives an estimate of the activation energy within HTST. In adaptions of these rate theories to magnetic systems [6-12], the magnitude of the magnetic vectors is either assumed to be constant as orientation changes, or it is treated as a fast variable obtained from self-consistency calculations for fixed values of the slow variables specifying orientation [13]. The energy surface of a system of $\mathrm{N}$ magnetic moments is then a function of $2 \mathrm{~N}$ degrees of freedom defining the orientation of the magnetic moments.

If all degrees of freedom in the system can be included within the harmonic approximation (no zero modes, i.e. degrees of freedom for which the energy is constant) HTST and Kramers estimate give an Arrhenius expression for the rate constant, $k(T)=f_{0} \exp \left[-\Delta E / k_{B} T\right]$ where $\Delta E$ is the energy difference between the relevant first order saddle point and the initial state minimum. The pre-exponential factor, $f_{0}$, often referred to as the attempt frequency, can be determined by calculating the eigenvalues of the Hessian matrix, the matrix of second derivatives of the energy with respect to the angles specifying the orientaiton of the magnetic moments, at the first order saddle point and at the initial state minimum [11].

While the activation energy for magnetic transitions has frequently been calculated, fewer estimates of the pre-exponential factor have been reported. Brown [6,7] estimated the pre-exponential factor for remagnetization 
transitions in a single domain, uniaxial magnetic particle to be on the order of $10^{9}-10^{12} \mathrm{sec}^{-1}$. The size and shape of the particle as well as the materials properties will affect the value. Experimental measurements by Wernsdorfer et al. [14] on $30 \mathrm{~nm}$ diameter Co nanoparticles gave an estimate of $4 \times 10^{9} \mathrm{sec}^{-1}$.

Recently, the HTST approach has been used to estimate the pre-exponential factor as well as the activation energy for various magnetic transitions. For remagnetization transitions in small Fe nanoislands on W(110), significantly larger values of the pre-exponential factor were obtained, ranging from $10^{13}$ to $10^{18} \mathrm{sec}^{-1}$, depending on the size and shape of the islands [15]. The higher range of values was found where the remagnetization occurs via formation of a temporary domain wall ('soliton' mechanism [2]). Experimental estimates for islands falling within a more limited range in shape and size are in close agreement with the calculated values [16]. An HTST estimate for larger permalloy islands used in kagome spin ice systems [17] gave a smaller value, $9.9 \times 10^{8} \mathrm{sec}^{-1}$ [18] while analysis of the experimental data had assumed a value of $10^{12} \mathrm{sec}^{-1}$ [17].

On the other hand, HTST calculations of magnetization reversal in a small Fe cluster at a tip interacting with an antiferromagnetic surface gave values in the range of $10^{12}$ to $10^{18} \mathrm{sec}^{-1}$ [19], while previous analysis of such measurements had assumed a pre-exponential factor of $10^{9} \mathrm{sec}^{-1}$ [20]. Since the values of the pre-exponential factor quoted here range over several orders of magnitude, it is clearly important to carry out calculations based on the energy landscape characterizing the magnetic system of interest, rather than just assuming some value $a$ priori.

Calculations of the value of the pre-exponential factor using HTST are in principle straightforward, even when the energy of the system is obtained from iterative self-consistent calculations [12,13]. Other examples of HTST calculations of transition rates include magnetic skyrmion annihilation in $\operatorname{CoPt}(111)$ films [21], skyrmion lifetime in narrow magnetic tracks [22] and the effect of impurities on skyrmion lifetime [23]. The dimensionality of the energy surface used for those calculations ranged from several hundreds up to tens of thousands. Good agreement has been found between HTST calculations and experimental measurements of lifetimes of both single and double kagome rings when the parameters in the calculations were determined from basic properties of a single island and no adjustment made to fit the data [18].

While robust methods are available for finding MEPs in complex magnetic systems [24,25], the visualization of the transition mechanism is in general a difficult task. To simplify the problem, one can consider a projection of the multidimensional surface on a two-dimensional surface, where the energy is given by a two-parameter function specifying the orientation of the magnetic moments in the system. This has, for example, been done in studies of magnetization reversals in an exchange spring magnet, where the mechanism was described as propagation of a temporary domain wall along the soft magnet toward the interface with the hard magnet and beyond [26]. The position and the width of the wall are the two parameters used to parametrize the projection of the energy surface.

A particularly simple and yet important example of a magnetic system is a single domain magnetic particle with shape anisotropy, possibly in the presence of a magnetic field. This system has been studied over a long period of time and is often referred to as a Stoner-Wohlfarth particle [27]. It is, for example, relevant for modeling of transitions in artificial spin ice systems which consist of arrays of magnetic islands on a solid surface. The energy of the magnetic particle can be described by two angles in a spherical coordinate system so the energy surface can be visualized easily. Previously, thermal effects on the lifetime of the magnetic states of such a particle have been studied using kinetic equations [7] and by calculations of the smallest non-vanishing eigenvalue of the Fokker-Plank equation [28]. Theoretical and experimental studies of the effect of temperature on dynamic hysteresis [29] have been carried out as well as studies of the effect of thermal fluctuations on magnetic anisotropy determinations [30]. However, the dependence of the pre-exponential factor in the rate constant on the size and shape of the magnetic particle as well as the materials properties is not well known and, as mentioned above, analysis of experimental data often relies on assumed values rather than accurate estimates.

In the present article, we report HTST calculations of the lifetime of magnetic states of a single domain magnetic particle with shape anisotropy with and without an applied magnetic field. The activation energy and pre-exponential factor for magnetic transitions are reported for various values of the anisotropy parameters. For the cases where the applied magnetic field is directed either along the anisotropy axis or perpendicular to it, explicit analytical expressions are obtained for the pre-exponential factor as well as the activation energy.

\section{Model}

We will consider a single domain magnetic island supported on a solid surface in the presence of an external magnetic field $\mathbf{H}$. The direction of the magnetic moment of the island is determined by two angles, $\theta$ and $\phi$, as shown in Fig. 1. The direction of the magnetic field will be characterized by angles $\theta_{H}$ and $\phi_{H}$.

The energy density can be written as the sum of an anisotropy term and a Zeeman term

$$
E / V=E_{\text {anis }} / V+E_{Z} / V \text {. }
$$




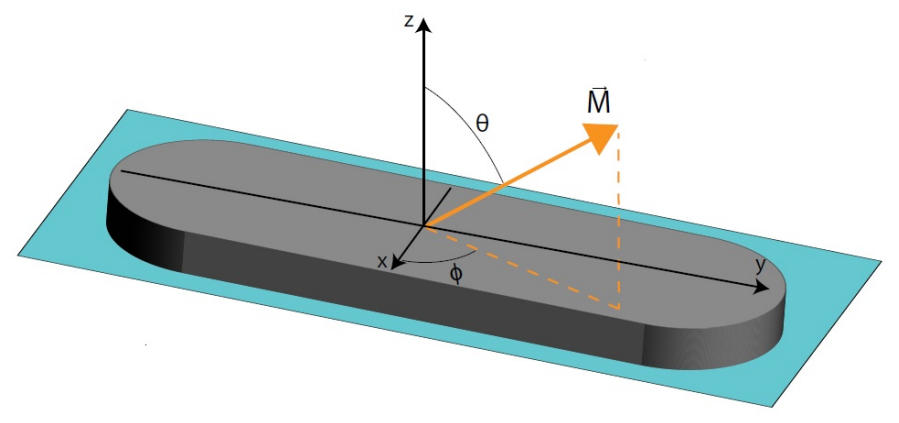

FIG. 1. Single domain magnetic island on a solid surface. $\vec{M}$ is the total magnetic moment of the island and its direction with respect to the anisotropy axis and the surface normal is given by the spherical polar angles $\theta$ and $\phi$.

Here, the anisotropy term can be written as

$$
E_{\text {anis }} / V=-K_{1} \sin ^{2} \theta \sin ^{2} \phi+K_{2} \cos ^{2} \theta,
$$

where $K_{1}$ and $K_{2}$ are anisotropy constants. For a permalloy island in a spin ice structure [17], $K_{1}>0$ and $K_{2}>0$ describe easy axis shape anisotropy and easy plane shape anisotropy, respectively. For CoPt islands [31,32], there is an intrinsic out of plane anisotropy and $K_{2}<0$ whereas $K_{1} \approx 0$ due to the round shape of the islands. The same system can be described by $K_{1}<0$ and $K_{2} \approx 0$ when the y-axis is chosen to be perpendicular to the island plane.

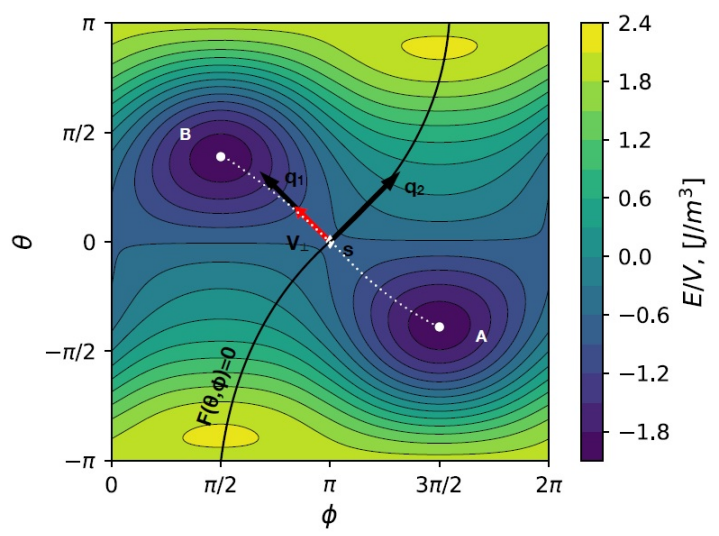

FIG. 2. Energy surface determined by eqs. (1-3) for parameters mimicking CoPt islands [31,32], $c=1.3$ and $h=1.8$ with magnetic field $H=1.33 \mathrm{MA} / \mathrm{m}$ in a direction given by $\theta_{H}=\pi / 10$ and $\phi_{H}=\pi / 2$ (the parameters used in the calculations are $M_{0}=836 \mathrm{kA} / \mathrm{m}, K_{1}=386 \mathrm{~kJ} / \mathrm{m}^{3}$ and $K_{2}=501.8 \mathrm{~kJ} / \mathrm{m}^{3}$ ). The local minima corresponding to the two stable states, A and B, are marked with white disks and the minimum energy path connecting them is shown with a white dotted line. The first order saddle point is marked with $\mathbf{s}$ and a dividing surface separating the A and $\mathrm{B}$ states, defined by $F(\theta, \phi)=0$, is shown with a black line. The velocity perpendicular to the dividing surface, $V_{\perp}$, near the saddle point is shown with a red arrow. The two normal mode vectors at the saddle point are shown as black arrows labeled $q_{1}$ and $q_{2}$

The Zeeman energy is given by the equation

$$
E_{Z} / V=-\mu_{0} H M_{0}\left(\sin \theta \sin \theta_{H} \sin \phi \sin \phi_{H}+\sin \theta \sin \theta_{H} \cos \phi \cos \phi_{H}+\cos \theta \cos \theta_{H}\right),
$$

where $M_{0}$ is the magnetization of the material and $H$ is the magnetic field strength.

An example energy surface determined by eq. (1-3) is depicted in Fig. 2. The values of parameters are $c \equiv K_{2} / K_{1}=1.3$ and $h \equiv \mu_{0} M_{0} H / 2 K_{1}=1.8$ (The parameters used in the calculations shown in Fig. 2 are $M_{0}=836 \mathrm{kA} / \mathrm{m}, K_{1}=386 \mathrm{~kJ} / \mathrm{m}^{3}$ and $K_{2}=501.8 \mathrm{~kJ} / \mathrm{m}^{3}$. In the calculations shown in Fig. 3 the value of $K_{2}$ is different, $116 \mathrm{~kJ} / \mathrm{m}^{3}$, to roughly correspond to CoPt islands [31,32]), and the magnetic field is chosen to have strength of $H=1.33 \mathrm{MA} / \mathrm{m}$ and direction given by $\theta_{H}=\pi / 10$ and $\phi_{H}=\pi / 2$. There are two local minima 
$\mathrm{A}$ and $\mathrm{B}$ on the energy surface corresponding to the stable magnetic states. The dividing surface separating the orientations that correspond to the $\mathrm{A}$ and $\mathrm{B}$ states can be represented as a continous curve $F(\theta, \phi)=0$. The MEP between the stable states was found using the geodesic nudged elastic band method [24], a generalization of the frequently used NEB method for atomic rearrangements [33]. The exact position of the first order saddle point was determined with the climbing image algorithm [34].

When the magnetic field is applied along or perpendicular to the easy axis and in the plane of the island, the energy surface is more symmetric and the values of the angles corresponding to stable states, the MEP and saddle point can be obtained analytically. Fig. 3 shows such a case for a magnetic field of $H=100 \mathrm{kA} / \mathrm{m}$. If the field is directed perpendicular to the easy axis (the y-axis), the local minima are at $\theta=\pi / 2$ and $\phi=\pi \pm \arccos h$. The value of $\theta$ is constant along the MEP and the saddle point is at $\theta=\pi / 2, \phi=\pi$. The activation energy is the same in this case for transitions in both directions $\Delta E / V=K_{1}(1-h)^{2}$. If the field is directed parallel to the easy axis, the local minima are at $\theta=\pi / 2$ and $\phi=\pi \pm \pi / 2$ and the saddle point is at $\theta=\pi / 2, \phi=\pi+\arcsin (-h)$.

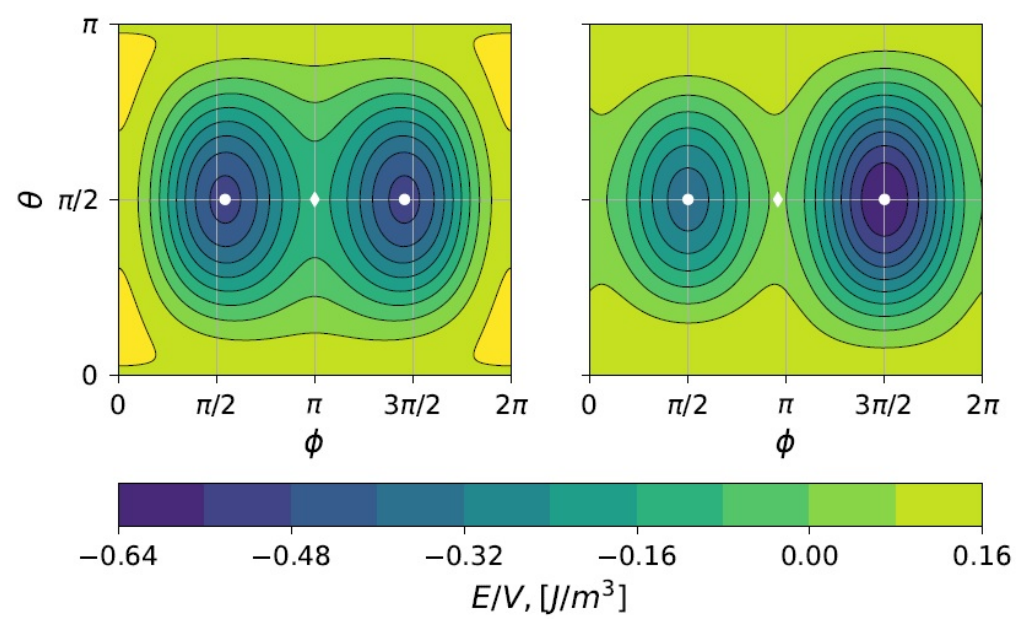

FIG. 3. Energy surface of a single domain magnetic island with parameter values the same as for Fig. 2 except that $c=0.3$ and the field strength is $H=100 \mathrm{kA} / \mathrm{m}$ (the parameters used in the calculations are $M_{0}=836 \mathrm{kA} / \mathrm{m}, K_{1}=386 \mathrm{~kJ} / \mathrm{m}^{3}$ and $K_{2}=116 \mathrm{~kJ} / \mathrm{m}^{3}$ ). The field is pointing perpendicular to the easy axis (the y-axis) on the left side, but parallel to the easy axis on the right side. The local minima and the first order saddle point are marked with white dots.

The two wells corresponding to the stable states are not equally deep. For transitions from the metastable state to the ground state, the activation energy is again $\Delta E / V=K_{1}(1-h)^{2}$. Note that the positions of the minima and saddle point as well as the activation energy do not depend on the in-plane anisotropy, $K_{2}$. The direction of the MEP is along $\theta=\pi / 2$, also independent of $K_{2}$. However, $K_{2}$ affects the shape of the energy surface, the variation of the energy with respect to $\theta$ when $\phi$ is constant, so the rate constant ends up being dependent on $K_{2}$, see below.

\section{Rate constant}

Within TST, the rate of transitions can be found as the product of the probability of reaching the transition state, a thin ribbon of configuration space around the dividing surface, $F(\theta, \phi)=0$, and the flux out of the transition state. The key approximation of TST is that a dynamical trajectory starting from the initial state only crosses the dividing surface once until the system thermalizes in the product state. Recrossing events during the traversal over the energy barrier are neglected. Dynamical trajectories started at the transition state, can be used to correct the TST estimate of the rate constant. The TST estimate of the rate constant is

$$
k^{T S T}=\frac{1}{Z} \iint_{S} e^{-E(\theta, \phi) / k_{B} T} V_{\perp} \sin \theta d \theta d \phi,
$$

where $S$ denotes the dividing surface and

$$
Z=\iint_{A} e^{-E(\theta, \phi) / k_{B} T} \sin \theta d \theta d \phi
$$


is the configuration integral for the system in the initial state, $\mathrm{A}$, and $V_{\perp}$ is the projection of the velocity vector, $\vec{V}$, onto the local normal of the dividing surface (that points to the final state B),

$$
V_{\perp}=\frac{\nabla \vec{F}}{\|\nabla \vec{F}\|} \cdot \vec{V}
$$

When the harmonic approximation is used, i.e. in HTST, the dividing surface is taken to be a hyperplane going through the first order saddle point with normal pointing along the MEP. A quadratic approximation of the energy surface in terms of $\theta$ and $\phi$ around the initial state minimum and the first order saddle point are used to estimate the activation energy and the flux out of the transition state. In order to eliminate mixed terms in the quadratic approximation, it is convenient to define a new coordinate system in terms of the eigenvectors of the Hessian matrix, the vibrational normal coordinates $q_{1}^{\beta}$ and $q_{2}^{\beta}$, where $\beta=s$ at the saddle point and $\beta=m$ at the initial state minimum

$$
E\left(q_{1}^{\beta}, q_{2}^{\beta}\right)=E\left(\theta_{\beta}, \phi_{\beta}\right)+\frac{1}{2}\left[\epsilon_{\beta 1}\left(q_{1}^{\beta}\right)^{2}+\epsilon_{\beta 2}\left(q_{2}^{\beta}\right)^{2}\right],
$$

where, $\epsilon_{\beta 1}$ and $\epsilon_{\beta 2}$ are the eigenvalues of the Hessian matrix at the saddle point or initial state minimum. The velocity is given by the Landau-Lifshitz equation, which in the vicinity of the first order saddle point, can be written in terms of the normal coordinates as

and

$$
\dot{q}_{1}^{s}=\frac{\gamma \epsilon_{s 2}}{V M_{0} \sin \theta_{s}} q_{2}^{s}
$$

$$
\dot{q}_{2}^{s}=-\frac{\gamma \epsilon_{s 1}}{V M_{0} \sin \theta_{s}} q_{1}^{s} .
$$

where $\gamma$ is the gyromagnetic ratio. If at the saddle point $\epsilon_{s 1}<0$ and $\epsilon_{s 2}>0$, then $V_{\perp}=\dot{q}_{1}^{s} \sin \left(\theta_{s}\right)$. Otherwise, if $\epsilon_{s 1}>0$ and $\epsilon_{s 2}<0$, then $V_{\perp}=\dot{q}_{2}^{s} \sin \theta_{s}$. The integral over the dividing surface in eqn. (4) needs to be carried out only for regions where $V_{\perp}>0$, i.e. for trajectories that are heading away from the initial state and towards the product state [11]. The velocity is zero at the saddle point, but non-zero contributions to the flux are obtained from one half of the hyperplanar dividing surface. Integration gives

$$
k^{H T S T}=\frac{\gamma \sqrt{\epsilon_{m 1} \epsilon_{m 2}}}{2 \pi M_{0} V \sin \theta_{m}} e^{-\Delta E / k_{B} T} .
$$

It is interesting to note that the pre-exponential factor does not depend on the Hessian at the saddle point, only at the initial minimum through the eigenvalues $\epsilon_{m 1}$ and $\epsilon_{m 2}$. This occurs for two-dimensional energy surfaces because $V_{\perp}>0$ is proportional to the one positive eigenvalue of the Hessian at the saddle point, but the same eigenvalue also appears in the denominator and thus cancels out. The pre-exponential factor also does not depend on the volume, $V$, because the eigenvalues of the Hessian at the minimum are proportional to the volume, and the volume also appears in the denominator, so it cancels out.

For the cases when the applied magnetic field is directed either parallel or perpendicular to the easy axis, an analytical expression for the rate constant in terms of the parameters characterizing the energy surface can be obtained. When the magnetic field is perpendicular to the easy axis, the result is

$$
k_{\perp}^{H T S T}=f_{0 \perp} e^{-\Delta E / k_{B} T}=\frac{\gamma K_{1} \sqrt{\left(1-h^{2}\right)(2 c+1)}}{\pi M_{0}} \exp \left[-\frac{V K_{1}}{k_{B} T}(1-h)^{2}\right],
$$

and when it is parallel to the easy axis, the result is

$$
k_{\|}^{H T S T}=f_{0 \|} e^{-\Delta E / k_{B} T}=\frac{\gamma K_{1} \sqrt{(1-h)(2 c-h+1)}}{\pi M_{0}} \exp \left[-\frac{V K_{1}}{k_{B} T}(1-h)^{2}\right] .
$$

Figure 4 shows the dependence of the pre-exponential factor, $f_{0}$, on the anisotropy parameters, $K_{1}$ and $K_{2}$ in the absence of an external magnetic field, when $M_{0}=200 \mathrm{kA} / \mathrm{m}$. A decrease of $K_{1}$ leads to a decrease of the pre-exponential factor but reduces at the same time the activation energy. These results show that a value of $10^{9}$ $\mathrm{Hz}$ as was assumed in refs. [31,32] gives reasonable approximation.

\section{Conclusion}

We have presented here simple equations that can be easily evaluated to estimate to estimate the rate constant for remagnetization transitions in a single domain magnetic particle where the transition occurs by uniform rotation. While the saddle point needs to be found in order to estimate the activation energy, $\Delta E$, the eigenvalues of the Hessian are not needed there, only at the initial state minimum. 


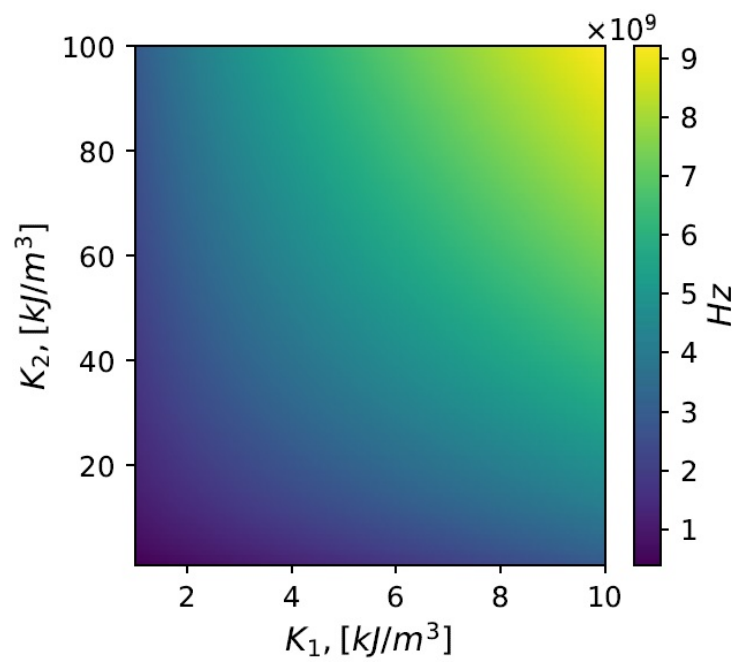

FIG. 4. Dependence of the pre-exponential factor on the anisotropy parameters $K_{1}$ and $K_{2}$ when the saturation magnetization is $M_{0}=200 \mathrm{kA} / \mathrm{m}$ and no external magnetic field is present, $H=0$

Especially simple analytical equations were obtained for the cases where the applied field is directed in either a parallel or perpendicular manner to the easy axis, or is absent. All that is needed to evaluate the rate constant in such cases is the saturation magnetization of the material and the two anisotropy constants. The rate constant turns out to be independent of the volume of the magnetic particle.

These results should be of value for theoretical estimates of magnetic transition rates in, for example, artificial spin ice systems, where previously, values of the pre-exponential factor have usually simply been assumed to have some value without relating to the basic properties of the individual islands. The approach presented here has already been shown to be accurate in calculations of the lifetime of single and double kagome rings [18].

For large enough islands compared with the strength of the magnetic interaction between the spins within the island, a uniform rotation is not the preferred transition mechanism, but rather a temporary domain wall. The results presented here do not apply to such situations. Calculations of Fe islands indicate that the pre-exponential factor can be substantially larger in such cases [15]. The reason may be that lower frequency modes then appear at the saddle point. Further analysis of the systematic trends in the pre-exponential factor in such cases as a function of the materials properties and the size and shape of the islands remains a topic of future studies.

Also, for low enough temperatures, quantum mechanical tunneling as opposed to the over-the-barrier mechanism considered here, will become the preferred transition mechanism. Recently, general equations for estimating the onset temperature for tunneling have been presented $[35,36]$ and can be used to give a lower bound on the temperature range for which the equations presented here remain reliable approximations.

\section{Acknowledgements}

This work was supported by the Icelandic Research Fund, the Academy of Finland (grant 278260) and the Government of the Russian Federation (grant 074-U01) and by grant 16-11-10330 of Russian Science Foundation.

\section{References}

[1] Coffey W.T., Garanin D.A. and McCarthy D.J. Crossover formulas in the Kramers theory of thermally activated escape rates - Application to spin systems.Advances in Chemical Physics, 2001, 117, P. 483.

[2] Braun H.B. Topological effects in nanomagnetism: from superparamagnetism to chiral quantum solitons. Advances in Physics, 2012, 61, P. 1.

[3] Wigner E. The transition state method. Trans. Faraday Soc., 1938, 34, P. 29-41.

[4] Kramers H.A. Brownian motion in a field of force and the diffusion model of chemical reactions. Physica, 1940, 7, P. $284-304$.

[5] Vineyard G.H. Frequency factors and isotope effects in solid state rate processes. J. Phys. Chem. Solids, 1957,3, P. 121.

[6] Brown Jr.W.F. Thermal fluctuations of a single-domain particle. Phys. Rev., 1963, 130, P. 1677.

[7] Brown Jr.W.F. Thermal Fluctuations of Fine Ferromagnetic Particles. IEEE Trans. Magn., 1979, MAG-15, P. 1196-1208.

[8] Braun H.-B. Kramers's rate theory, broken symmetries and magnetization reversal. J. Appl. Physics, 1994, 76, P. 6310-6315.

[9] Visscher P.B., Zhu R. Low-dimensionality energy landscapes: Magnetic switching mechanisms and rates. Physica B, 2012, 407, P. 13401344.

[10] Fiedler G., Fidler J., Lee J., Schrefl T., Stamps R.L., Braun H.B. and Suess D., Direct calculation of the attempt frequency of magnetic structures using the finite element method. J. Appl. Phys., 2012, 111, P. 093917(7). 
[11] Bessarab P.F., Uzdin V.M. and Jónsson H. Harmonic Transition State Theory of Thermal Spin Transitions. Phys. Rev. B, 2012, 85, P. 184409(4).

[12] Bessarab P.F., Uzdin V.M. and Jónsson H. Potential Energy Surfaces and Rates of Spin Transitions. Z. Phys. Chem., 2013, 227, P. 15431557.

[13] Bessarab P.F., Uzdin V.M. and Jónsson H. Calculations of magnetic states and minimum energy paths of transitions using a noncollinear extension of the Alexander-Anderson model and a magnetic force theorem. Phys. Rev. B, 2014, 89, P. 214424(12).

[14] W. Wernsdorfer, E. Bonet Orozco, K. Hasselbach, A. Benoit, B. Barbara, N. Demoncy, A. Loiseau, H. Pascard and D. Mailly. Experimental evidence of the Néel-Brown model of magnetization reversal. Phys. Rev. Lett., 1997, 78, P. 1791.

[15] Bessarab P.F., Uzdin V.M. and Jónsson H. Size and Shape Dependence of Thermal Spin Transitions in Nanoislands. Phys. Rev. Lett., 2013, 110, P. 020604(5)

[16] Krause S., Herzog G., Stapelfeldt T., Berbil-Bautista L., Bode M., Vedmedenko E. Y., Wiesendanger R. Magnetization reversal of nanoscale islands: How size and shape affect the Arrhenius Pprefactor. Phys.Rev. Lett., 2009, 103, P. 127202(4).

[17] Farhan A., Derlet P.M., Kleibert A., Balan A., Chopdekar R.V., Wyss M., Anghinolfi L., Nolting F., Heyderman L.J. Exploring hyper-cubic energy landscapes in thermally active finite artificial spin-ice systems. Nature Physics, 2013, 9, P. 375(8).

[18] Liashko S.Y., Uzdin V.M. and Jónsson H. The effect of temperature and external field on transitions in elements of kagome spin ice. New J. Phys., 2017, (accepted) DOI: 10.1088/1367-2630/aa8b96.

[19] Ivanov A., Bessarab P.F., Uzdin V.M. and Jónsson H. Magnetic exchange force microscopy: Theoretical analysis of induced magnetization reversals. Nanoscale, 2017, 9, P. 13320-13325.

[20] Schmidt R., Schwarz A., Wiesendanger R. Magnetization switching utilizing the magnetic exchange interaction. Phys. Rev. B, 2012, 86, P. 174402(6).

[21] Lobanov I.S., Jónsson H. and Uzdin V. M. Mechanism and activation energy of magnetic skyrmion annihilation obtained from minimum energy path calculations. Phys. Rev. B , 2016, 94, P. 174418(7).

[22] Uzdin V.M., Potkina M.N., Lobanov I.S., Bessarab P.F., Jónsson H. The effect of confinement and defects on the thermal stability of skyrmions. Physica B, 2017, (in press), DOI; 10.1016/j.physb.2017.09.040.

[23] Uzdin V.M., Potkina M.N., Lobanov I.S., Bessarab P.F., Jónsson H. Energy surface and lifetime of magnetic skyrmions. J. Magn. Magn. Mat., (accepted). Manuscript available at https://arxiv.org/pdf/1707.00124.pdf.

[24] Bessarab P.F., Uzdin V.M. and Jónsson H. Method for finding mechanism and activation energy of magnetic transitions, applied to skyrmion and antivortex annihilation. Comp. Phys. Commun., 2015, 196, P. 335-347.

[25] Bessarab P. F. Comment on "Path to collapse for an isolated Néel skyrmion". Phys. Rev. B , 2017, 95, P. 136401(2).

[26] Moskalenko M. , Bessarab P.F., Uzdin V.M. and Jónsson H. Qualitative Insight and Quantitative Analysis of the Effect of Temperature on the Coercivity of a Magnetic System. AIP Advances, 2016, 6, P. 025213(8).

[27] Stoner E.C., Wohlfarth E.P. A mechanism of magnetic hysteresis in heterogeneous alloys. Phil. Trans. Roy. Soc. A, 1948, 240, P. 599-642.

[28] Coffey W.T, Crothers D.S.F., Dormann J.L., Kalmykov Y.P., Kennedy E.C., Thermally activated relaxation time of a single domain ferromagnetic particle subjected to a uniform field at an oblique angle to the easy axis: Comparison with experimental observations. Phys. Rev. Lett., 1998, 80, P. 5655-5568.

[29] Poperechny I.S., Raikher Yu.L., Stepanov V.I. Dynamic magnetic hysteresis in single-domain particles with niaxial anisotropy. Phys.Rev. $B, 2010,82$, P. 174423(14).

[30] Franco V., Conde A. Thermal effects in a StonerWohlfarth model and their influence on magnetic anisotropy determination. J. Magn. Magn. Mat., 2004, 278, P. 28-38.

[31] De Vries J., Bolhuis T., Abelmann L. Temperature dependence of the energy barrier and switching field of sub-micron magnetic islands with perpendicular anisotropy. New J. Phys., 2017, (accepted) DOI:10.1088/1367-2630/aa8082.

[32] Engelen J.B.C., Delalande M., le Febre A.J., Bolhuis T., Shimatsu T., Kikuchi N., Abelmann L., Lodder J.C. Thermally induced switching field distribution of a single CoPt dot in a large array. Nanotechnology, 2010, 21, P. 035703(7).

[33] Jónsson H., Mills G., Jacobsen K.W. Classical and Quantum Dynamics in Condensed Phase Simulations, edited by Berne B.J., Ciccotti G., Coker D.F. (World Scientific, Singapore, 1998), P. 385-404.

[34] Henkelman G., Uberuaga B.P., Jónsson H. A climbing image nudged elastic band method for finding saddle points and minimum energy paths. J. Chem. Phys., 2000, 113, P. 9901-9904.

[35] Vlasov S., Bessarab P.F., Uzdin V.M. and Jónsson H. Classical to quantum mechanical tunneling mechanism crossover in thermal transitions between magnetic states. Faraday Discuss., 2016, 195, P. 93-109.

[36] Vlasov S., Bessarab P.F., Uzdin V.M. and Jónsson H. Calculations of the onset temperature for tunneling in multispin systems. Nanosystems: Physics, Chemistry, Mathematics, 2017, 8, P. 454-461. 